\title{
Free Vibration Analysis of Shear Deformable Small-Scale Tubes
}

\author{
${ }^{* 1}$ Reza Aghazadeh \\ *1Department of Mechatronics Engineering, University of Turkish Aeronautical Association, Turkey
}

\begin{abstract}
This study presents an approach for investigating free vibration problem of small-scale tubes based on modified couple stress theory in conjunction with higher order shear deformation tube model. The size effect is captured through utilization of a length scale parameter involved in modified couple stress theory. A newly developed refined tube model is employed to satisfy friction-free conditions on inner and outer surfaces of micro-tubes. Hamilton's principle is used as a variational technique for derivation of governing system of equations. For axial vibrations, an analytical procedure is conducted, while for transverse vibrations differential quadrature method is used as a numerical technique. The correctness of numerical results are verified through comparisons made with results which are available in the literature for limiting cases. The analyses reveal the effects of size and transverse shear deformation on the natural frequencies of micro-tubes.
\end{abstract}

Key words: Small-scale tubes, modified couple stress theory, higher order shear deformation theory, length scale parameter, free vibration analysis

\section{Introduction}

Tubes/pipes are among mechanical components, which are extensively used in structural systems. Along with the recent advances in the fields of micro- and nano- mechanical systems (MEMS and NEMS) and manufacturing technologies, small-sized tubes have attracted considerable attention. The rapidly growing applications of small-scale tubes has urged many researchers to investigate mechanical behavior of these structures. It is experimentally evidenced that classical elasticity is incapable of predicting the size-dependent behavior of micro-structures [1, 2]. Hence, some researchers have put forward novel higher order continuum theories. Among these nonclassical theories, couple stress theories [3, 4], strain gradient theories [1, 5], nonlocal elasticity [6], nonlocal strain gradient theory [7] and surface elasticity [8] are commonly used by researchers.

In most of the applications, tubes can be modeled as beams with tubular cross-sections. Bending, buckling and free vibration problems of tubular micro-beams have intensively been addressed by many researchers. In studies conducted by Chakraverty and Jena [9], Civalek and Demir [10], Mercan and Civalek [11] and Shafiei et al. [12] mechanics of micro- and nano-tubes have been investigated based on Eringen's non-local elasticity theory. Modified couple stress theory (MCST) by Yang et al. [4] has been employed to analyze mechanical behavior of small-sized pipes in a number of papers [13-15]. Zhen et al. [16] and Mehralian et al. [17] investigated the free vibration problem of nano-tubes by utilizing nonlocal strain gradient theory.

In all studies mentioned in the foregoing paragraph, as well as most studies available in the

*Corresponding author: Address: Department of Mechatronics Engineering, University of Turkish Aeronautical Association, 06790, Ankara - TURKEY. E-mail address: raghazadeh@thk.edu.tr, Phone: +903125896132 
literature, Euler-Bernoulli and Timoshenko beam models are generally used to express the displacement field. However, the transverse shear stress is neglected in Euler-Bernoulli model, and Timoshenko beam theory considers a constant shear stress through the thickness of the tube, which are both strictly simplifying assumptions. Therefore, there is a need for a refined model to satisfy stress-free conditions in the inner and outer surfaces of tubes. Although there are variety of higher order shear deformable models for beams possessing rectangular cross-sections [18, 19], the number of refined annular tube models are few. By taking the shear deformation effects into account Zhang and Fu [20] established a higher order tube model (HOTM), appropriate for tubular beams. They conducted bending, free vibrations and wave propagation analyses based on newly developed model and compared their results with conventional theories. Based on HOTM, some researches regarding mechanics of macro- and small-scale tubes have been carried out. In some of these studies higher order shear deformable model is used in conjunction with nonlocal elasticity to put forward an accurate size-dependent analyses technique for tubes [21, 22]. In order to investigate vibrations of buckled functionally graded (FG) tubes in thermal environment, Babaei and Reza Eslami [23] employed the modified couple stress theory and higher order tube model. Based on refined tube model, Zhong et al. [24] studied bending and vibration problems of FG tubes considering the temperature effects.

In the current study, governing equations and associated boundary conditions for free vibration problem of micro-tubes are derived in accordance with MCST and HOTM. Displacement field of the annular micro-tube is expressed in a unified way to be able to produce numerical results corresponding to three different beam theories, which are Euler-Bernoulli, Timoshenko, and higher order shear deformable tube model. Equation system of motion is solved by means of differential quadrature method (DQM). Presented numerical results illustrate influences of scale effects and different tube models upon natural frequencies of micro-tubes.

\section{Formulation}

Fig. 1 shows the configuration and geometrical parameters of a micro-tube having an inner radius $r_{i}$, an outer radius $r_{o}$ and a length $L . u_{1}, u_{2}$ and $u_{3}$ denote the displacements of any point at time $t$ along $x_{1}, x_{2}$ and $x_{3}$ directions, respectively, and can be expressed in a unified form as given below:

$u_{1}\left(x_{1}, x_{3}, t\right)=u\left(x_{1}, t\right)-x_{3} w_{, x_{1}}+f\left(x_{2}, x_{3}\right) \gamma\left(x_{1}, t\right)$

$u_{2}\left(x_{1}, x_{3}, t\right)=0$

$u_{3}\left(x_{1}, x_{2}, x_{3}, t\right)=w\left(x_{1}, t\right)$

$u$ and $w$ designate the displacement of any point on horizontal middle surface along $x_{1}$ and $x_{3}$ directions, respectively, "," stands for partial derivative, $\gamma$ is the transverse shear strain of any point on the neutral axis and can be related to bending rotation $\phi$ through

$\gamma\left(x_{1}, t\right)=w_{, x_{1}}\left(x_{1}, t\right)+\phi\left(x_{1}, t\right)$,

Through-the-thickness distribution of transverse shear strain and stress is characterized by shape function $f$. Different tube models, namely Euler - Bernoulli tube model (EBTM), Timoshenko tube model (TTM) and higher order tube model (HOTM) [20] can be derived by utilizing the following shape functions: 
EBTM: $\quad f\left(x_{2}, x_{3}\right)=0$

TTM: $\quad f\left(x_{2}, x_{3}\right)=x_{3}$

HOTM: $f\left(x_{2}, x_{3}\right)=x_{3}+\frac{x_{3}}{\left(r_{o}^{2}+r_{i}^{2}\right)}\left(\frac{r_{o}^{2} r_{i}^{2}}{r^{2}}-\frac{r^{2}}{3}\right)$

Note that the shear stress is neglected in EBTM; TTM considers a constant distribution profile for transverse shear; and a nonlinear pattern is achieved for shear strain and stress using HOTM.
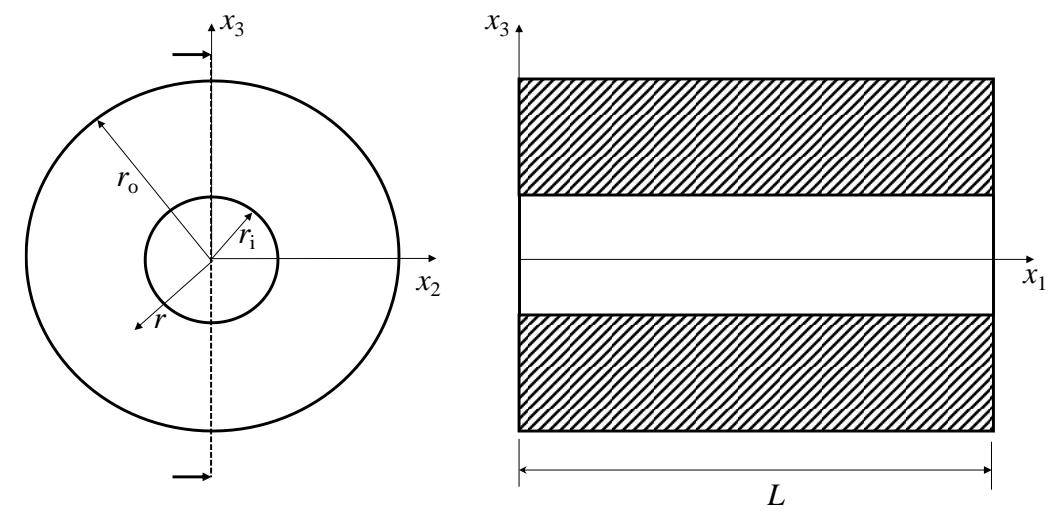

Fig. 1. Tube configuration

Partial differential equations of motion and boundary conditions governing the dynamic problem of the micro-tube are derived by using Hamilton's principle. It postulates that

$\delta \int_{t_{1}}^{t_{2}}(K-U) d t=0$.

Note that for free vibration analysis, the work done by external forces are disregarded. $U$ and $K$ in Eq. (4) are total strain energy and kinetic energy, respectively.

According to modified couple stress theory (MCST) [4], strain energy $U$ of the micro-tube is written as:

$U=\frac{1}{2} \int_{\Omega}\left(\sigma_{i j} \varepsilon_{i j}+m_{i j} \chi_{i j}\right) d V$,

where $\sigma_{i j}$ and $\varepsilon_{i j}$ are classical stress and strain components; $\chi_{i j}$ is symmetric curvature tensor; $m_{i j}$ is the higher order stress tensor associated with $\chi_{i j}$; and $\Omega$ is occupied volume.

The deformation gradients in Eq. (5) are defined by

$\varepsilon_{i j}=\frac{1}{2}\left(u_{i, j}+u_{j, i}\right)$,

$\chi_{i j}=\frac{1}{2}\left(e_{i p q} \varepsilon_{q j, p}+e_{j p q} \varepsilon_{q i, p}\right)$.

$e_{i j k}$ represents alternating tensor, and $\delta_{i j}$ is Kronecker delta. Constitutive relations are written as 
$\sigma_{i j}=2 \mu \varepsilon_{i j}+\lambda \delta_{i j} \varepsilon_{k k}$

$m_{i j}=2 \mu l^{2} \chi_{i j}$

$\lambda$ and $\mu$ are Lame's constants given by

$\lambda=\frac{E v}{(1+v)(1-2 v)}, \quad \mu=\frac{E}{2(1+v)}$

where $E$ is modulus of elasticity and $v$ is Poisson's ratio. $l$ in Eq. (7.b) is the material length scale parameter characterizing the small-scale effect.

Kinetic energy is of the forms:

$K=\frac{1}{2} \int_{\Omega} \rho\left\{\left(\frac{\partial u_{1}}{\partial t}\right)^{2}+\left(\frac{\partial u_{2}}{\partial t}\right)^{2}+\left(\frac{\partial u_{3}}{\partial t}\right)^{2}\right\} d V$,

where $\rho$ is mass density.

Introducing expressions for $U$ and $K$ and associated relations into Hamilton's principle leads to the following system of governing partial differential equations:

$\delta u: A_{11} \frac{\partial^{2} u}{\partial x_{1}^{2}}=I_{1} \frac{\partial^{2} u}{\partial t^{2}}$,

$\delta w:$

$$
\begin{aligned}
& \left(2 F_{22}-D_{11}-F_{33}+F_{152}-A_{552}-\frac{1}{4} F_{552}-\frac{1}{4} F_{772}\right) \frac{\partial^{4} w}{\partial x_{1}^{4}}+k_{s}\left(F_{55}+F_{77}\right) \frac{\partial^{2} w}{\partial x_{1}^{2}} \\
& +\frac{1}{4}\left(F_{662}+F_{882}-2 F_{682}+4 F_{992}\right) \frac{\partial^{2} w}{\partial x_{1}^{2}}+\left(F_{22}-F_{33}+\frac{1}{2} F_{152}-\frac{1}{4} F_{552}-\frac{1}{4} F_{772}\right) \frac{\partial^{3} \phi}{\partial x_{1}^{3}} \\
& +\left(k_{s} F_{55}+k_{s} F_{77}+\frac{1}{4}\left(F_{662}+F_{882}-2 F_{682}+4 F_{992}\right)\right) \frac{\partial \phi}{\partial x_{1}} \\
& =\left(2 I_{5}-I_{3}-I_{6}\right) \frac{\partial^{4} w}{\partial x_{1}^{2} \partial t^{2}}+I_{1} \frac{\partial^{2} w}{\partial t^{2}}+\left(I_{5}-I_{6}\right) \frac{\partial^{3} \phi}{\partial x_{1} \partial t^{2}},
\end{aligned}
$$

$\delta \phi:$

$$
\begin{aligned}
& \left(F_{33}-F_{22}+\frac{1}{4} F_{552}-\frac{1}{2} F_{152}+\frac{1}{4} F_{772}\right) \frac{\partial^{3} w}{\partial x_{1}^{3}}-k_{s}\left(F_{55}+F_{77}\right) \frac{\partial w}{\partial x_{1}} \\
& -\frac{1}{4}\left(F_{662}+F_{882}-2 F_{682}+4 F_{992}\right) \frac{\partial w}{\partial x_{1}}+\left(F_{33}+\frac{1}{4}\left(F_{552}+F_{772}\right)\right) \frac{\partial^{2} \phi}{\partial x_{1}^{2}} \\
& +\left(-k_{s}\left(F_{55}+F_{77}\right)-\frac{1}{4}\left(F_{662}+F_{882}-2 F_{682}+4 F_{992}\right)\right) \phi=\left(I_{6}-I_{5}\right) \frac{\partial^{3} w}{\partial x_{1} \partial t^{2}}+I_{6} \frac{\partial^{2} \phi}{\partial t^{2}} .
\end{aligned}
$$

and the boundary conditions read

$$
\delta u=0 \quad \text { or } \quad A_{11} \frac{\partial u}{\partial x_{1}}=0
$$




$$
\begin{aligned}
& \delta w=0 \text { or }\left(2 F_{22}-D_{11}-F_{33}+F_{152}-A_{552}-\frac{1}{4} F_{552}-\frac{1}{4} F_{772}\right) \frac{\partial^{3} w}{\partial x_{1}^{3}}+k_{s}\left(F_{55}+F_{77}\right) \\
& +\frac{1}{4}\left(F_{662}+F_{882}-2 F_{682}+4 F_{992}\right) \frac{\partial w}{\partial x_{1}}-\left(F_{33}-F_{22}+\frac{1}{4} F_{552}-\frac{1}{2} F_{152}+\frac{1}{4} F_{772}\right) \frac{\partial^{2} \phi}{\partial x_{1}^{2}} \\
& +\left(k_{s}\left(F_{55}+F_{77}\right)+\frac{1}{4}\left(F_{662}+F_{882}-2 F_{682}+4 F_{992}\right)\right) \phi=\left(2 I_{5}-I_{3}-I_{6}\right) \frac{\partial^{3} w}{\partial x_{1} \partial t^{2}}-\left(I_{6}-I_{5}\right) \frac{\partial^{2} \phi}{\partial t^{2}}, \\
& \delta \frac{\partial w}{\partial x_{1}}=0 \text { or }\left(D_{11}+F_{33}-2 F_{22}+A_{552}+\frac{1}{4} F_{552}-F_{152}+\frac{1}{4} F_{772}\right) \frac{\partial^{2} w}{\partial x_{1}^{2}} \\
& +\left(F_{33}-F_{22}+\frac{1}{4} F_{552}-\frac{1}{2} F_{152}+\frac{1}{4} F_{772}\right) \frac{\partial \phi}{\partial x_{1}}=0, \\
& \delta \phi=0 \text { or }\left(F_{33}-F_{22}+\frac{1}{4} F_{552}-\frac{1}{2} F_{152}+\frac{1}{4} F_{772}\right) \frac{\partial^{2} w}{\partial x_{1}^{2}}+\left(F_{33}+\frac{1}{4} F_{552}+\frac{1}{4} F_{772}\right) \frac{\partial \phi}{\partial x_{1}}=0 .
\end{aligned}
$$

$k_{s}$ here is the shear correction factor and is taken as unity for HOTM and for Timoshenko beam model when used for annular cross-section is given as follows [25]

$k_{s}=\frac{6\left(\eta^{2}+1\right)^{2}(1+v)^{2}}{\left(7+12 v+4 v^{2}\right)\left(\eta^{4}+1\right)+\left(34+48 v+16 v^{2}\right) \eta^{2}}$

where $\eta=r_{i} / r_{o}$. The stiffness and inertia parameters appeared in Eqs. (10) and (11) are as follows

$$
\begin{aligned}
& \left\{A_{11}, D_{11}, F_{22}, F_{33}\right\}=\frac{E(1-v)}{(1+v)(1-2 v)} \int_{A}\left\{1, x_{3}^{2}, x_{3} f, f^{2}\right\} d A \\
& \left\{F_{55}, F_{77}\right\}=\mu \int_{A}\left\{f_{, x_{3}}^{2}, f_{, x_{2}}^{2}\right\} d A \\
& \left\{A_{552}, F_{552}, F_{152}, F_{662}, F_{992}, F_{682}, F_{882}, F_{772}\right\} \\
& =\mu l^{2} \int_{A}\left\{1, f_{, x_{3}}^{2}, f_{, x_{3}}, f_{, x_{3} x_{3}}^{2}, f_{, x_{2} x_{3}}^{2}, f_{, x_{3} x_{3}} f_{, x_{2} x_{2}}, f_{, x_{2} x_{2}}^{2}, f_{, x_{2}}^{2}\right\} d A \\
& \left\{I_{1}, I_{3}, I_{5}, I_{6}\right\}=\rho \int_{A}\left\{1, x_{3}^{2}, x_{3} f, f^{2}\right\} d A
\end{aligned}
$$

\section{Numerical Solution}

The system comprising governing differential equations and boundary conditions is numerically solved by employing differential quadrature method (DQM). In this method, $m^{\text {th }}$ derivative of a function is approximated by a weighted sum of functional values at all grid points. For a tube that is discretized in longitudinal direction by a number of nodes $N$, the $m^{\text {th }}$ derivative of a function $z(x, t)$ with respect to $x$ at a point $x_{i}$ is written as:

$\left.\frac{\partial^{m} z(x, t)}{\partial x^{m}}\right|_{x=x_{i}}=\sum_{j=1}^{N} c_{i j}^{(m)} z\left(x_{j}, t\right), \quad i=1,2, \ldots, N$.

where $c_{i j}^{(m)}$ are the weighting coefficients. 
In order to conduct free vibration analysis dynamic displacement vector is defined by $\mathbf{d}=\mathbf{d}^{*} \mathrm{e}^{\mathrm{i} \omega t}$

where $\omega$ represents the natural frequency and $\mathbf{d}^{*}$ is the vibration mode shape vector consisting of dynamic displacement vectors of field variables

$\mathbf{d}^{*}=\left\{\left\{u_{p}^{*}\right\}^{\mathrm{T}},\left\{w_{p}^{*}\right\}^{\mathrm{T}},\left\{\phi_{p}^{*}\right\}^{\mathrm{T}}\right\}^{\mathrm{T}}$, for $p=1,2, \ldots, N$

Substitution of Eq. (15) into governing equations and boundary conditions leads to the following eigenvalue problem

$\left\{\mathbf{K}-\omega^{2} \mathbf{M}\right\} \mathbf{d}^{*}=\mathbf{0}$,

where $\mathbf{K}$ is stiffness matrix and $\mathbf{M}$ is mass matrix derived from inertia terms.

Although natural frequencies associated with the axial vibrations, $\omega_{\text {axial }}$, can be computed using the numerical procedures explained above, by further inspecting governing equations and boundary conditions, it can be understood that axial vibration, characterized by Eqs. (10.a) and (11.a), is fully decoupled from transverse ( $w$ and $\phi$ ) vibration. In addition, the relations for axial dynamics can be solved analytically, which, for simply supported micro-tube, gives the following closed-form expression for $\omega_{\text {axial }}$

$\omega_{\text {axial }}=\frac{n \pi}{L} \sqrt{\frac{A_{11}}{I_{1}}}$

where $n$ is the axial vibration mode number.

\section{Numerical Results}

A simply supported micro-tube is considered to carry out numerical analyses. Material properties are similar to those of stainless steel with $E=201.04 \mathrm{GPa}, v=0.3$ and $\rho=8166 \mathrm{~kg} / \mathrm{m}^{3}$. Unless otherwise mentioned the geometrical dimensions are taken as $r_{o}=10 \mu \mathrm{m}, r_{i} / r_{o}=1 / 2, L=20 r_{o}$.

The natural frequencies are non-dimensionalized through following relation $\bar{\omega}=\omega r_{o} \sqrt{\frac{\rho}{\mu}}$

In Table 1 first dimensionless natural frequencies are compared to those given by Zhang and $\mathrm{Fu}$ [20]. Classical elasticity theory is used in conjunction with HOTM and TTM to produce the results provided in Table 1. The results are in excellent conformity with those of Zhang and $\mathrm{Fu}$ [20]. 
Table 1. Comparisons of first dimensionless natural frequencies computed based on classical elasticity theory, $l=0$.

\begin{tabular}{ccccccc}
\hline \multirow{2}{*}{$L / r_{o}$} & \multirow{2}{*}{$r_{i} / r_{o}$} & \multicolumn{2}{c}{ HOTM } & & \multicolumn{2}{c}{ TTM } \\
\cline { 3 - 4 } \cline { 6 - 6 } & & Present & Zhang and Fu [20] & & Present & Zhang and Fu [20] \\
\hline $20 / 3$ & $1 / 3$ & 0.167 & 0.167 & & 0.168 & 0.168 \\
\hline $25 / 3$ & $2 / 3$ & 0.121 & 0.121 & & 0.123 & 0.123 \\
\hline $200 / 21$ & $19 / 21$ & 0.104 & 0.104 & & 0.105 & 0.105 \\
\hline
\end{tabular}

Fig. 2 shows the variations of first dimensionless transverse natural frequencies with outer radius to length scale parameter ratios $r_{o} / l$. The results are generated based on Timoshenko theory, TTM, and refined tube model, HOTM. Also, the results of nonclassical MCST and classical elasticity theories can be compared. Note that $r_{o} / l$ is altered by changing the $l$-value and keeping $r_{o}$ as constant. The natural frequencies are seen to be an increasing function of the length scale parameter l. Although the results predicted by TTM and HOTM are very close to each other, a slightly smaller natural frequencies are computed by TTM. Another finding from Fig. 2 is that, as $r_{o}$ becomes sufficiently larger than length scale parameter $l$, the natural frequencies produced by MCST converge to those of conventional continuum theory. It is also worth mentioning that, axial vibration is not affected by change in the value of $l$ and the first dimensionless axial natural frequency $\bar{\omega}_{\text {axial }}=0.2939$ for all values of $r_{o} / l$.

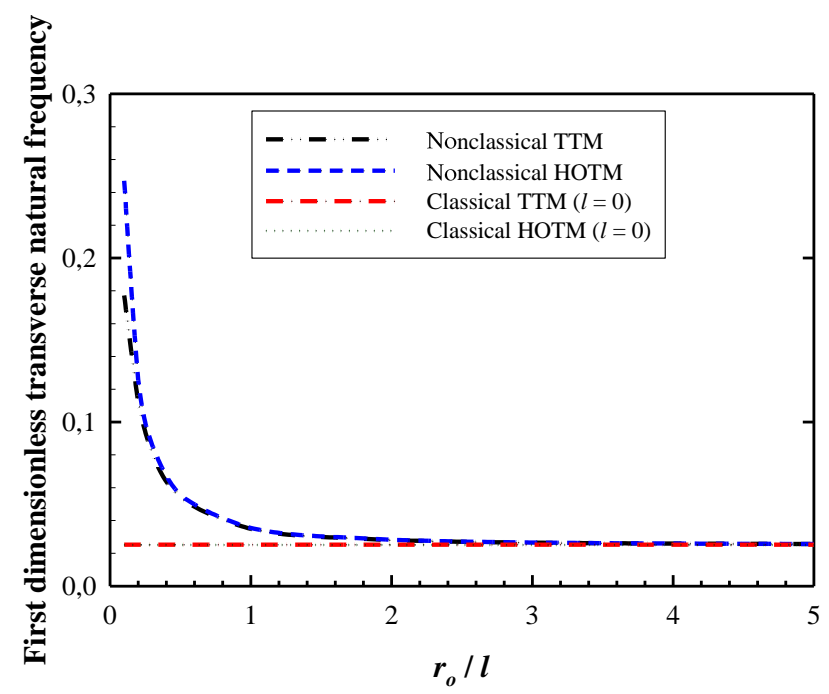

Fig. 2. First dimensionless transverse natural frequencies

Provided in Table 2 are the first five dimensionless natural frequencies evaluated in different values of $r_{o} / l$. In this table, the frequencies belonging to axial vibrations are distinguished by "**". Similar to findings of Fig. 2, increase in value of length scale parameter results in corresponding increase in the transverse natural frequencies. However, a change in $r_{o} / l$ has no effect on axial vibration frequencies. Generally, the first three natural frequencies in micro-tubes belong to transverse motion, when classical theory is used, i. e. when the size is sufficiently larger than micro-scale. Further inspection on Table 2 shows that introducing size effects via utilization of a length scale 
parameter leads to a stiffer micro-tube in transverse direction which may cause axial vibrations to be dominant.

Table 2. First five dimensionless natural frequencies computed using HOTM.

\begin{tabular}{cccccc}
\hline & \multicolumn{5}{c}{ Mode No. } \\
\cline { 2 - 5 }$r_{o} / l$ & 1 & 2 & 3 & 4 & 5 \\
\hline 0.1 & 0.2471 & $0.2939^{*}$ & $0.5877^{*}$ & $0.8816^{*}$ & 0.9749 \\
\hline 0.5 & 0.0554 & 0.2179 & $0.2939^{*}$ & 0.4787 & $0.5877^{*}$ \\
\hline 1.5 & 0.0302 & 0.1164 & 0.2487 & $0.2939^{*}$ & 0.4165 \\
\hline$l=0$ & 0.0251 & 0.0939 & 0.1923 & $0.2939^{*}$ & 0.3082 \\
\hline
\end{tabular}

*Dimensionless axial natural frequencies.

\section{Conclusion}

A new model for free vibration analysis of micro-tubes is developed based on modified couple stress theory. By employing a general displacement field, it is possible to retrieve micro-tube models based on different beam theories such as Euler-Bernoulli, Timoshenko and higher order shear deformation. An analytical solution procedure is developed for axial vibrations while DQM is utilized to conduct parametric analyses regarding transverse dynamics.

The influence of length scale parameter upon natural frequencies is investigated by means of numerical results. It is observed that $l$ has significant effect on mechanical responses of microtubes, such that, higher value of length scale parameter results in stiffer tube. As $l$ gets sufficiently smaller than geometric dimensions of tube, i.e. when tube thickness is so larger than length scale parameter, small-scale effect vanishes and results converge to those of classical theories.

The differences observed between the natural frequency results of TTM and HOTM postulates that employing a refined higher order tube model is indispensable for an accurate prediction of mechanical responses. The frequencies predicted by TTM are slightly smaller than those generated by HOTM, because, TTM overestimates shear stresses which leads to a less stiff micro-tube. HOTM gives a proper shear stress distribution profile, and hence, results in more accurate evaluation of natural frequencies.

\section{References}

[1] Lam DCC, Yang F, Chong ACM, Wang J, Tong P. Experiments and theory in strain gradient elasticity. Journal of the Mechanics and Physics of Solids 2003; 51(8): 1477-1508.

[2] Andrew WM, Jonathan SC. Role of material microstructure in plate stiffness with relevance to microcantilever sensors. Journal of Micromechanics and Microengineering 2005; 15(5): 1060 . 
[3] Mindlin RD, Tiersten HF. Effects of couple-stresses in linear elasticity. Archive for Rational Mechanics and Analysis 1962; 11(1): 415-448.

[4] Yang F, Chong ACM, Lam DCC, Tong P. Couple stress based strain gradient theory for elasticity. International Journal of Solids and Structures 2002; 39(10): 2731-2743.

[5] Aifantis EC. Strain gradient interpretation of size effects. International Journal of Fracture 1999; 95(1-4): 299-314.

[6] Eringen AC. Nonlocal polar elastic continua. International Journal of Engineering Science 1972; 10(1): 1-16.

[7] Lim CW, Zhang G, Reddy JN. A higher-order nonlocal elasticity and strain gradient theory and its applications in wave propagation. Journal of the Mechanics and Physics of Solids 2015; 78: 298-313.

[8] Gurtin ME, Weissmuller J, Larche F. The general theory of curved deformable interfaces in solids at equilibrium. Philosophical Magazine A 1998; 178: 1093-1109.

[9] Chakraverty S, Jena SK. Free Vibration of Single Walled Carbon Nanotube Resting on Exponentially Varying Elastic Foundation. Curved and Layered Structures 2018; 5(1): 260.

[10] Civalek Ö, Demir C. A simple mathematical model of microtubules surrounded by an elastic matrix by nonlocal finite element method. Applied Mathematics and Computation 2016; 289: 335-352.

[11] Mercan K, Civalek Ö. Buckling analysis of Silicon carbide nanotubes (SiCNTs) with surface effect and nonlocal elasticity using the method of HDQ. Composites Part B: Engineering 2017; 114: 34-45.

[12] Shafiei N, Mirjavadi SS, Afshari BM, Rabby S, Hamouda AMS. Nonlinear thermal buckling of axially functionally graded micro and nanobeams. Composite Structures 2017; 168: 428439.

[13] SafarPour H, Ghadiri M. Critical rotational speed, critical velocity of fluid flow and free vibration analysis of a spinning SWCNT conveying viscous fluid. Microfluidics and Nanofluidics 2017; 21(2): 22.

[14] Şimşek M. Dynamic analysis of an embedded microbeam carrying a moving microparticle based on the modified couple stress theory. International Journal of Engineering Science 2010; 48(12): 1721-1732.

[15] Yang T-Z, Ji S, Yang X-D, Fang B. Microfluid-induced nonlinear free vibration of microtubes. International Journal of Engineering Science 2014; 76: 47-55.

[16] Zhen Y-X, Wen S-L, Tang Y. Free vibration analysis of viscoelastic nanotubes under longitudinal magnetic field based on nonlocal strain gradient Timoshenko beam model. 
Physica E: Low-dimensional Systems and Nanostructures 2019; 105: 116-124.

[17] Mehralian F, Tadi Beni Y, Karimi Zeverdejani M. Nonlocal strain gradient theory calibration using molecular dynamics simulation based on small scale vibration of nanotubes. Physica B: Condensed Matter 2017; 514: 61-69.

[18] Şimşek M, Reddy JN. Bending and vibration of functionally graded microbeams using a new higher order beam theory and the modified couple stress theory. International Journal of Engineering Science 2013; 64: 37-53.

[19] Aghazadeh R, Cigeroglu E, Dag S. Static and free vibration analyses of small-scale functionally graded beams possessing a variable length scale parameter using different beam theories. European Journal of Mechanics - A/Solids 2014; 46: 1-11.

[20] Zhang P, Fu Y. A higher-order beam model for tubes. European Journal of Mechanics A/Solids 2013; 38: 12-19.

[21] She G-L, Yuan F-G, Ren Y-R, Liu H-B, Xiao W-S. Nonlinear bending and vibration analysis of functionally graded porous tubes via a nonlocal strain gradient theory. Composite Structures 2018; 203: 614-623.

[22] She G-L, Yuan F-G, Ren Y-R, Xiao W-S. On buckling and postbuckling behavior of nanotubes. International Journal of Engineering Science 2017; 121: 130-142.

[23] Babaei H, Reza Eslami M. Size-dependent vibrations of thermally pre/post-buckled FG porous micro-tubes based on modified couple stress theory. International Journal of Mechanical Sciences 2020; 180: 105694.

[24] Zhong J, Fu Y, Wan D, Li Y. Nonlinear bending and vibration of functionally graded tubes resting on elastic foundations in thermal environment based on a refined beam model. Applied Mathematical Modelling 2016; 40(17): 7601-7614.

[25] Hutchinson JR. Shear Coefficients for Timoshenko Beam Theory. Journal of Applied Mechanics 2000; 68(1): 87-92. 